\title{
Wireless Control of Vehicle Mirror System Using Head Movement and PIC Microcontroller
}

\author{
${ }^{1}$ Nidal F. Shilbayeh and ${ }^{2}$ Mahmoud Z. Iskandarani \\ ${ }^{1}$ Faculty of Computer Science and Information Technology, Applied Science University \\ P.O. Box 41, Post Code 11931, Amman, Jordan \\ ${ }^{2}$ Faculty of Science and Information Technology, Al-Zaytoonah Private University of Jordan \\ P.O. Box 911597, Post Code 11191, Amman, Jordan
}

\begin{abstract}
A PIC controlled IR system for the control car mirror system movement is designed and implemented. The designed and built system allows the side view mirrors to be adjusted based on the driver head movement. The infrared based head tracking system maps a predetermined coordinates for head movements and results in a triangularly computable geometry which is fed to the PIC based controlling system. This will cause the vehicle mirror movement via carefully selected miniature motors. The system takes blind spots and their angles into consideration
\end{abstract}

Key words: Automotive, Head Gesture, PIC Microcontroller, Wireless, Infrared, Control

\section{INTRODUCTION}

Gesture recognition is a complex task which involves many aspects such as motion modeling, motion analysis, pattern recognition, machine learning, and neural fuzzy systems. Gestures are expressive and meaningful body motions used in daily life as means of communication where a computer based automatic recognition system is necessary for interpretation and signal control in an interactive and dynamic environment... In such an environment body motion can be defined as a sequence of states in a configurable space, which can be modeled, based on the following principles:

* Static start and end position

* Smooth transition forward and backward

To recognize gesture patterns based on time series (state transitions). Traditionally the Hidden Macrov Model (HMM), Artificial Neural Network (ANN) and Dynamic Programming Matching Model (DB Matching) have been used in conventional research [1-2].

In this study we describe a new movement that detect head movement in real time which is more difficult as head gestures could be a short and fast moves particularly as we are applying it in an automotive environment such as car vehicles where the driver needs to produce a control signal during a short period of time. For this reason a non conventional PIC based control system employing IR transmitter receiver with colonized arrays is used to eliminate any interference that might be caused if an alternative video based system with conventional interpretation techniques is implemented [3-4].

Background: Using technologies and know-how from mechanical engineering, electronics, IT and telecommunications, there is a need to have on-board systems with capability to operate in harsh environments. Such systems are designed to gather and process information in order to carry out the actions necessary to achieve their designated functions. To accomplish their objective, in other words, to know how to "act", on-board systems operate using reaction models. The Brain of the system reacts to impulses, picked up by the sensors, and transmits information and orders to the actuators, of the system. All of the components, materials and software for these systems satisfy requirements concerning size, sturdiness, energy consumption, and immunity to external disturbances; the key words are: security, reliability, quality, safety, real-time, autonomy, and servo assistance. On-board electronic systems control the movements of rockets, satellites and planes, and car vehicles.

In an increasingly competitive environment, the parts manufacturer, specialized in automotive electronics, is continuing to grow, constantly reinforcing its technological edge. Among the latest innovations, the year 2000 marked the appearance of the communicating vehicle, with the integration of a WAP telephone in the car, opening new development perspectives for onboard electronic telemetric. The mobile Internet has joined forces with the car. The new concept car, also integrates biometry, with fingerprint sensors for user recognition and restitution of personalized adjustments 
(position of seat, steering wheel, or rear view mirrors), tactile screens, an on-board camera and an entirely redesigned dashboard for improved ergonomics and pleasant driving [5-6].

System Description: The micro-controller based design incorporates an Infrared sensor using photodiode LED, which serves as an interface between the sensor and the actuator incorporated into the side mirror. The Designed system consists of following parts:

Driver's Head Motion Detector: IR Motion Detector: IR (Infra Red) photodiode sensor circuit attached on the side-view mirror. The circuit below shows how to use simple infrared components for object detection. The unique aspect of this project is its adaptive ability to adjust the power-output levels of the infrared LED to compensate for variations in ambient lighting conditions as shown in Fig. 1.

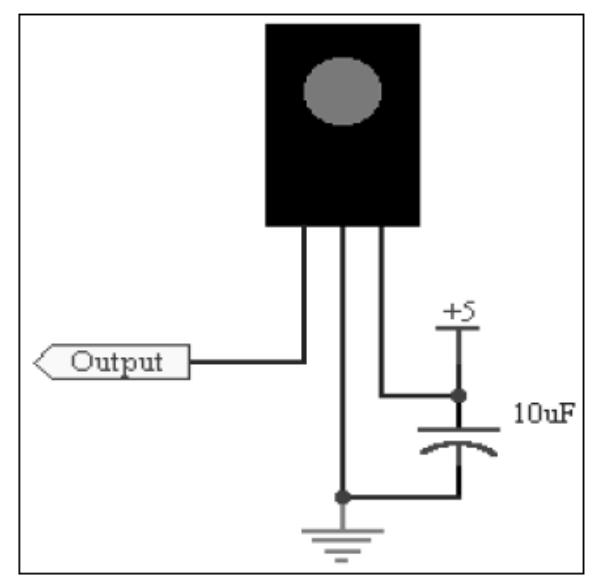

Fig. 1: IR Motion Detector Circuit

The infrared detector module used for this work is the $38 \mathrm{kHz}$ Panasonic PNA4602M. In dark lighting conditions the detector module increases the gain of the internal amplifier circuit. In bright lighting conditions, the internal gain is reduced. This reduction in gain is an attempt to reduce the possibility of false output pulses on the detectors output stage caused by light sources not associated with the intended data signal. The big effect of high ambient light levels is reduction of the overall operating distance of the IR LED, and decreased IR detector module sensitivity.

Most common infrared detectors require the data signal to arrive in the form of a modulated signal. The modulation component is referred to as the carrier. One stage of the detectors internal circuitry consists of a band-pass filter. The job of the band-pass filter is to reject light energy or un-wanted data signals not modulated at the band-pass filter center frequency.

This helps keep the output stage of the detector from emitting false-pulses or dark-bits, and is a simple method for discriminating between ambient light or noise, and the intended data signal. For this application we need to sample the logic output at the detectors output stage.

To produce a change in the detectors output stage we need:

* An infrared LED

* A means of modulating [turning ON \& OFF] the infrared LED at the carrier frequency

The output stage of this IR detector includes an internal pull-up resistor. In the resting state [no data or carrier being received] this pull-up resistor holds the IR detector modules output-pin at logic 1 . The logic value [ 1 or 0$]$ on the output-pin of the infrared detector can be sampled by the PIC microcontroller using a simple circuit. The output of the IR detector module can be connected to any available I/O-pin on the PIC. For this design PortB, bit \#7 is used. Below is the code to detect a valid carrier being received by the IR detector module, and light an LED connected to PortB, bit \#0. The LED in this case is used for visual verification that the infrared carrier or signal is being received by the IR detector module.

$\begin{array}{ll}\text { IF PortB.7 }=0 \text { THEN } & \text { 'If a logic } 0 \text { is detected, then } \\ \text { HIGH PortB.0 } & \text { 'Tum ON the LED } \\ \text { ELSE } & \text { 'If not, then } \\ \text { LOW PortB.0 } & \text { 'Tum OFF the LED } \\ \text { ENDIF } & \end{array}$

The output of the detector module is held at logic 1 by the internal pull-up resistor until infrared energy modulated at the band-pass frequency $(38 \mathrm{kHz})$ strikes the face of the detector module. Once the modulated signal is detected, the output of the detector goes to logic 0 (ground), and indicates to the PIC that infrared energy modulated at the carrier frequency is being received by the detector module.

For this application, we are mainly interested in knowing when the IR energy from the infrared LED is reflected back onto the face of the IR detector module. The code above causes the LED connected to PortB, bit \#0 to turn ON when the reflected IR energy strikes the face of the detector causing the detectors output-pin to transition from logic 1 to 0 as shown in Fig. 2.

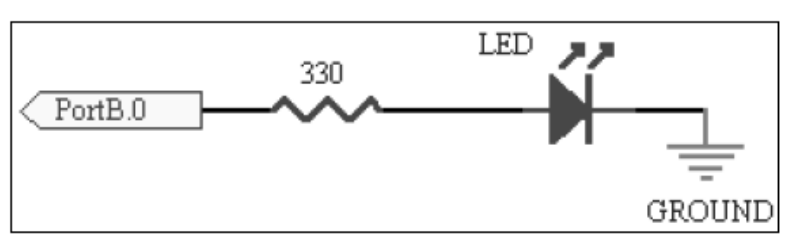

Fig. 2: PIC Controlled Visual Detecting Circuit

When PortB.7 $=0$ (38kHz carrier is detected), the LED is turned $\mathrm{ON}$. When no valid carrier is detected, the 
LED is turned OFF. We now have a way to detect objects in the path of the IR LED close enough to reflect the IR energy back onto the detector, and an indicator to verify detection. The $10 \mathrm{uF}$ capacitor used in the detector circuit helps to stabilize the output. Weak reflections from the infrared LED, and other sources will cause very small fluctuations on the output stage of the detector module causing the indicator LED to flash quickly as an object moves closer to the detector module. The capacitor acts as a filter, and ensures that only a strong reflected infrared signal produces the desired output of logic 0 by absorbing the small negative noise spikes. Without the 10uF capacitor, a very fast ON / OFF fluctuations of the LED connected to PortB. 0 will occur.

The infrared LED used for this work is the Lumex OED-EL-8L. This IR LED has a $40^{\circ}$ beam-angle, and is capable of emitting $180 \mathrm{~mW}$ of IR energy at a peak wavelength of $940 \mathrm{~nm}$ as shown in Fig. 3 .

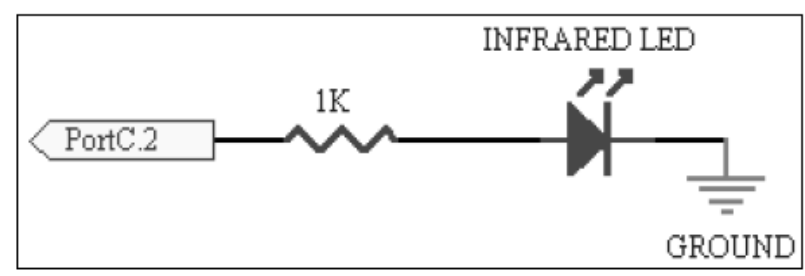

Fig. 3: PIC Controlled IR Detecting Circuit

The $40^{\circ}$ beam-angle of the OED-EL-8L infrared LED can be narrowed by placing a small piece of heat-shrink tubing over the LED lens. For this work the $40^{\circ}$ beamangle is narrowed for more precise distance measurements, and to eliminate side target signal reflections as shown in Fig. 4.

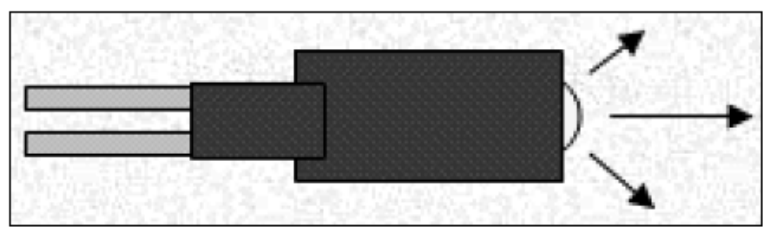

Fig. 4: Control of Beam Spread Using External Sleeve

The black heat-shrink tubing covering the LED lens keeps IR energy from being emitted side-ways, and affecting forward measurements. The heat-shrink on the LED leads keeps IR energy from exiting the rear of the lens element. Infrared energy is projected forward, and then reflected back onto the surface of the detector module positioned directly behind the infrared emitter. The actual placement of the heat-shrink is covering more of the LED, but for the purpose of illustration - I have shown the LED lens protruding slightly from the heat-shrink. You can adjust the heat-shrink to cover more or less as necessary, but try to eliminate IR emissions from the rear, and side of the LED.
Effectively measuring distances, and detecting objects directly in front of the emitter will be more precise with a narrow beam-angle. By controlling the output power we can adapt to changing environmental conditions, and ambient light sources or noise.

The infrared detector modules internal circuitry will attempt to increase or decrease the gain of the detection circuit proportional to the amount of ambient light striking the surface of the detector. High levels of ambient light will force the internal automatic gaincontrol to reduce the gain of the detection amplifier, thereby requiring a much stronger infrared signal striking the surface of the IR detector for valid detection. Increased gain due to lower ambient light levels or dark conditions means the IR detector is operating with increased sensitivity, and hence will detect a much weaker IR signal than in a disturbed or noisy environment. However, by using the PWM module on the PIC this issue is resolved. The PIC microcontroller has an onboard "hardware" PWM module. This PWM module offers true multi-tasking operation, and provides an excellent way to modulate the infrared LED at the required band-pass frequency of the detector module. Multi-tasking is the ability to process multiple tasks simultaneously. Once the PIC registers are configured to produce our required PWM frequency, and the PWM output is turned $\mathrm{ON}$, the designed code can go about other tasks while the PIC PWM hardware continues to emit the carrier frequency without further intervention. This multi-tasking ability allows modulation of the IR LED at the required carrier frequency while performing other tasks such as:

* Sample the output-pin of the IR detector (without stopping the PWM carrier)

* Take real-time ambient light readings with a CDS photocell

* Adjust the PWM duty-cycle to control IR output power

Using the information obtained from readings of ambient light levels, we can adjust the output power of the IR LED by varying the duty-cycle of the PWM signal. This adaptive technique allows compensating for changing environmental conditions, and adapting as necessary to maintain a constant infrared energy output required for reliable "reflective" IR sensing under variable lighting conditions. Measuring ambient light levels is simple with the PicBasic compiler POT command. Below is the simple circuit used for measuring light levels.

The POT command charges the capacitor through the CDS photocell and then measures the time it takes for the capacitor to discharge. The resulting value is then used to adjust the IR LED output power to compensate for changing light levels present on the infrared detector module as shown in Fig. 5. 


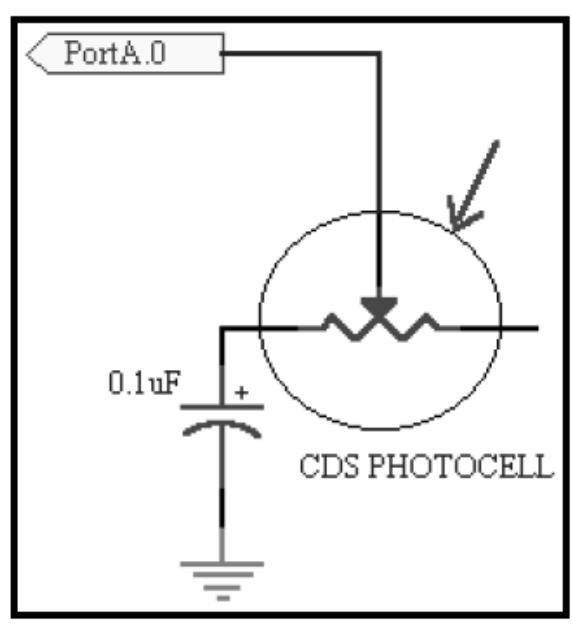

Fig. 5: Light Compensation Circuit

The duty-cycle of the PWM signal can easily be modified by placing different values into the CCPR $1 \mathrm{~L}$ register. The high-time of the duty cycle will turn ON the LED connected to PortC.2. The low cycle will turn the LED OFF. The Peak-Current through the infrared LED will be:

$\mathrm{I}_{\mathrm{P}}=\frac{V_{C C}-V_{L E D}}{R_{S}}$

$\mathrm{I}_{\mathrm{P}} \quad=$ The peak pulsed current through the infrared

LED

$\mathrm{V}_{C C}=$ The operating voltage. Here it is +5 VDC

$V_{L E D}=$ The voltage drop across the infrared LED.

For the OED-EL-8L this is $1.7 \mathrm{~V}$

$\mathrm{R}_{S} \quad=$ The current-limiting series resistor used

between the PortC. 2 V/O-pin, and the infrared LED

The result is $3.3 \mathrm{~mA}$ peak pulsed current as shown below:

$\frac{5 \mathrm{~V}-1.7 \mathrm{~V}}{1 \mathrm{~K}}=\frac{3.3 \mathrm{~V}}{1 \mathrm{~K}}=0.0033$

With a duty-cycle of $100 \%$, the maximum peak current through the infrared LED would be approximately $3.3 \mathrm{~mA}$.

For detecting objects at distances up to $6^{11}$ or more, the OED-EL-8L provides sufficient output power operating at a mere 264-660uA. This is excellent for battery powered robotics applications, and just about anything with low power requirements. It's not always necessary to use the standard $10-20 \mathrm{~mA}$ ratings used with most common LED circuits for all applications.

For even further power-savings, supply power to the infrared detector module using a single I/O-pin, and turn OFF the PWM module when not being used. The average current delivered to the infrared LED is important. Going over the average current will, in most cases, degrade or completely destroy the LED. This application stays well below the average current rating of the OED-EL-8L. Here's how:

$$
\begin{aligned}
& I_{\text {AVG }}=I_{P} X D_{F} \\
& I_{\text {AVG }}=\text { Average LED current } \\
& I_{P}=\text { Peak pulsed LED curren } \\
& D_{F}=\text { Duty factor or PWM [duty cycle] }
\end{aligned}
$$

With a maximum peak current of $3.3 \mathrm{~mA}$, and a duty cycle varying from $8-20 \%$, the average current range for the infrared LED used in this application is:

$3.3 \mathrm{~mA} \times 20 \%=660 \mathrm{uA} \mathrm{MAX}$, and $3.3 \mathrm{~mA} \times 8 \%=$ 264uA MIN

Driving the Mirrors: The mirrors are driven using a Stepper Motor or DC servo, which is a built-in motor to position the mirrors. A Servo is a small device that has an output shaft. This shaft can be positioned to specific angular positions by sending the servo a coded signal. As long as the coded signal exists on the input line, the servo will maintain the angular position of the shaft. As the coded signal changes, the angular position of the shaft changes. In practice, servos are used in radiocontrolled airplanes to position control surfaces like the elevators and rudders. They are also used in radiocontrolled cars, and of course, robots. Servos are extremely useful in robotics. The motors are small, as you can see by the picture above, have built in control circuitry, and are extremely powerful for their size. The servomotor has some control circuits and a potentiometer (a variable resistor) that is connected to the output shaft. This pot allows the control circuitry to monitor the current angle of the servomotor. If the shaft is at the correct angle, then the motor shuts off. If the circuit finds that the angle is not correct, it will turn the motor the correct direction until the angle is correct. The output shaft of the servo is capable of traveling somewhere around 180 degrees. Usually, it's somewhere in the 210 degree range, but it varies by manufacturer. A normal servo is used to control an angular motion of between 0 and 180 degrees. A normal servo is mechanically not capable of tuming any farther due to a mechanical stop built on to the main output gear.

The amount of power applied to the motor is proportional to the distance it needs to travel. So, if the shaft needs to turn a large distance, the motor will run at full speed. If it needs to turn only a small amount, the motor will run at a slower speed. This is called proportional control. 


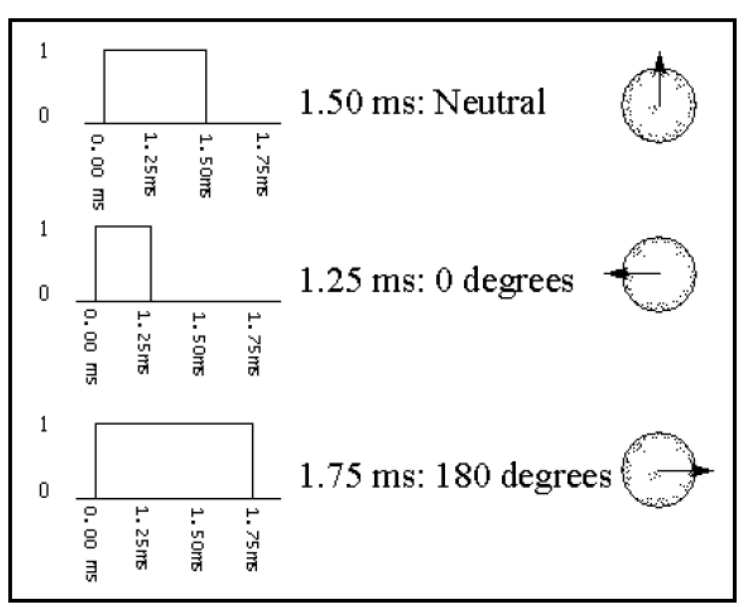

Fig. 6: Stepper Motor Positions

The control wire is used to communicate the angle. The angle is determined by the duration of a pulse that is applied to the control wire. This is called Pulse Coded Modulation. The servo expects to see a pulse every 20 milliseconds ( 0.02 seconds). The length of the pulse will determine how far the motor turns. A 1.5millisecond pulse, for example, will make the motor turn to the 90-degree position (often called the neutral position). If the pulse is shorter than $1.5 \mathrm{~ms}$, then the motor will turn the shaft to closer to 0 degrees. If the pulse is longer than $1.5 \mathrm{~ms}$, the shaft turns closer to 180 degrees as shown in Fig. 6.

As we can see in the picture, the duration of the pulse dictates the angle of the output shaft (shown as the green circle with the arrow). Note that the times here are illustrative and the actual timings depend on the motor manufacturer. The principle, however, is the same.

Servomotors are used in most RC cars, boats, helicopters and planes. They are often used to control sensitive adjustments such as steering, but have many other uses in robotics and positioning control systems.

Servomotors are basically geared down de motors with positional feedback control, allowing for accurate positioning of the rotor, with a range of 90 degrees. They can also be modified to allow for continuous rotation.

Servomotors have three wires; usually red, black and white. The red wire is for +VDC, the black for ground and the white is for position control. This control signal is a variable-width pulse, which can be varied from 1 to $2 \mathrm{~ms}$. The pulse width controls the rotor position.

A $1.0 \mathrm{~ms}$ pulse rotates the shaft all the way counterclockwise. A $1.5 \mathrm{~ms}$ pulse puts the rotor at neutral $(0$ degrees), and a $2.0 \mathrm{~ms}$ pulse will position the shaft all the way clockwise. The pulse is sent to the servo at a frequency of approximately $50 \mathrm{~Hz}$. The relationship between the pulse width and the rotor position can be seen in Fig. 7.

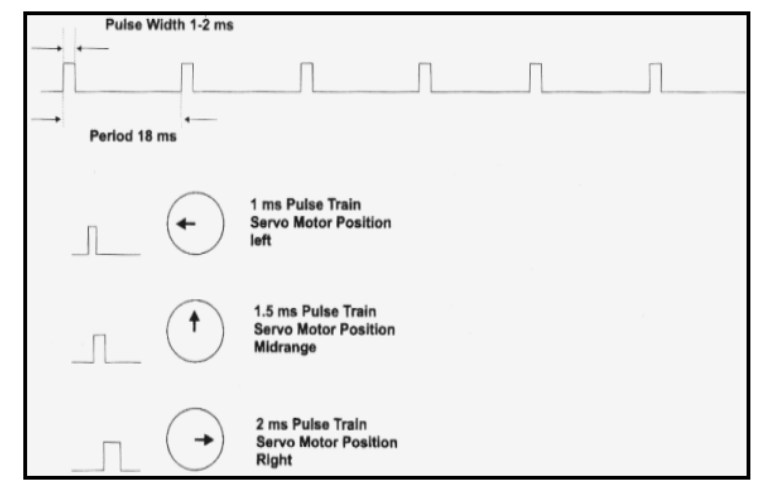

Fig. 7: Relationship Between the Pulse Width and the Rotor Position

Our program will allow controlling the direction of the servo according to the IR detector connected to port B1. The overall system schematic diagram is shown in Fig.8, which shows a low input to port B1; hence, the servomotor will not turn. When it is changed to high, it will turn one way until the detector output is low or the servo get to the last position. If it was in its last position, it will change direction and proceeds until the $\mathrm{o} / \mathrm{p}$ of the detector is low. The 16F84 PIC Microcontroller serves as the electronic interface between the sensor and the motor. It contains $1 \mathrm{~K}$ words of FLASH program memory, 68 bytes of data RAM, and 64 bytes of data EEPROM. While this seems like an extremely limited amount of code and data space, the PIC's incredibly compact code makes the most of it. 1024 instruction word memory actually means 1024 instructions, no less. Even immediate-mode instructions, where an operand is part of the instruction itself, takes only one memory location, as do CALL and GOTO instructions. There even exists a single-chip implementation of a TCP/IP stack and HTTP server written for a $16 \mathrm{~F} 84$.

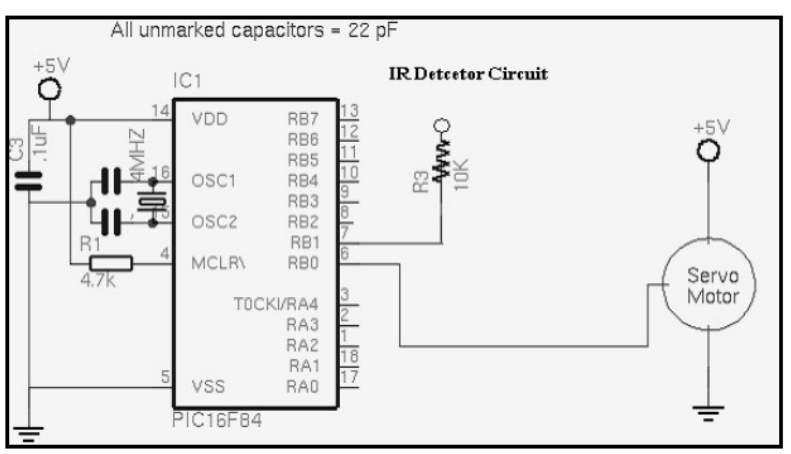

Fig. 8: The Overall PIC Based Control System

The seemingly odd 14-bit instruction word length is a direct result of the internal architecture of the processor itself. In the case of the $16 \mathrm{~F} 84$ or $16 \mathrm{C} 711$, we need 13 bits just to address all of program memory. In the case of the smaller 16C54 with only 512 words of program 
memory and 25 bytes of RAM, we can get by with a 12-bit instruction word -- which is exactly what the 16C54 uses. Conversely, with more memory we would use a longer instruction word, like the 16 bits in the 18 Cxxx family.

The PIC is also a non-micro coded design. In larger processors, each binary machine language instruction often is "executed" by a series of micro code steps. While this is a great approach for building large, complex processors with a wide range of instructions, it also leads to great complexity and takes up a lot of real estate. The PIC uses the instruction word itself, decoded by logic gates as it is read from program memory, to control the flow of data through the chip.

\section{RESULTS AND DISCUSSION}

Figure 9 shows the overall arrangement and positioning of sensors in the driver's cabinet for system tests and verification; with Fig. 10 illustrating the mathematical tracing curve for the driver's head movement that was implemented in our PIC controlled system.

The mathematical curve representing head gesture takes into consideration an area whereby any movement of the head will not initiate mirrors movement (dead zone). This is essential, as the mirrors need not to move for simple head movements that result from natural human responses to scenes on the road or to the head movements of passengers close by to the driver of the vehicle. The limits for the dead zone is determined and implemented into the software controlling algorithms as a result of calculations on the vehicle internal space[7-9].

Two algorithms are used to test our PIC controlled vehicle mirror system:

* Mirror Control Algorithm

* Light Compensation Algorithm

The first algorithm test the control signal applied to Servo Motors that adjust the position of the vehicle Mirrors. This carried out via controlling voltage, and frequency signals sent to these motors. Consequently it will result in an adjustment of mirror angels. The algorithm takes into account signal failure as it continues to check the progress of mirror movement and ensures the stability of these mirrors in its new position [10-13].

The second algorithm serves to ensure the correct operation of the designated system regardless of the outside light effect that might interfere with the sensors positioned inside the vehicle. Hence, it assures the right movements of the mirrors during daytime or night time. Both algorithms work together using our programmable PIC controller.

As the above algorithms work hand in hand on processing the detected signals resulting from the driver

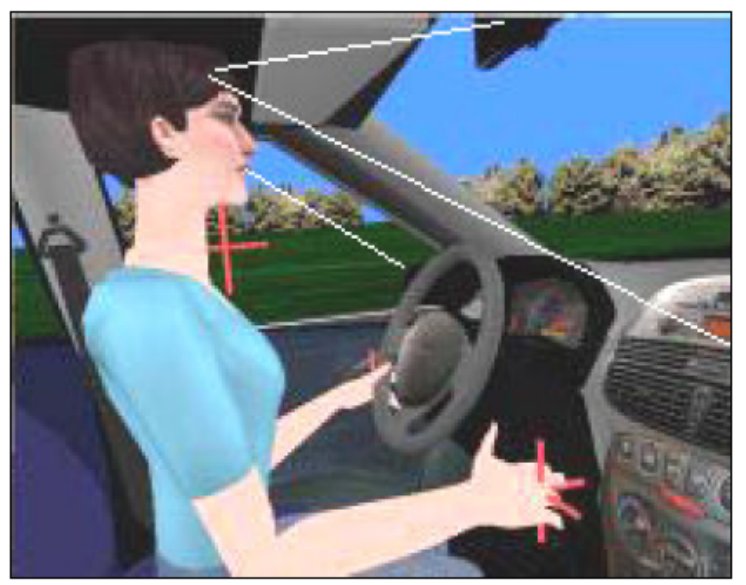

Fig. 9: New Driver Cabinet Design for Head Movent Mirror Control

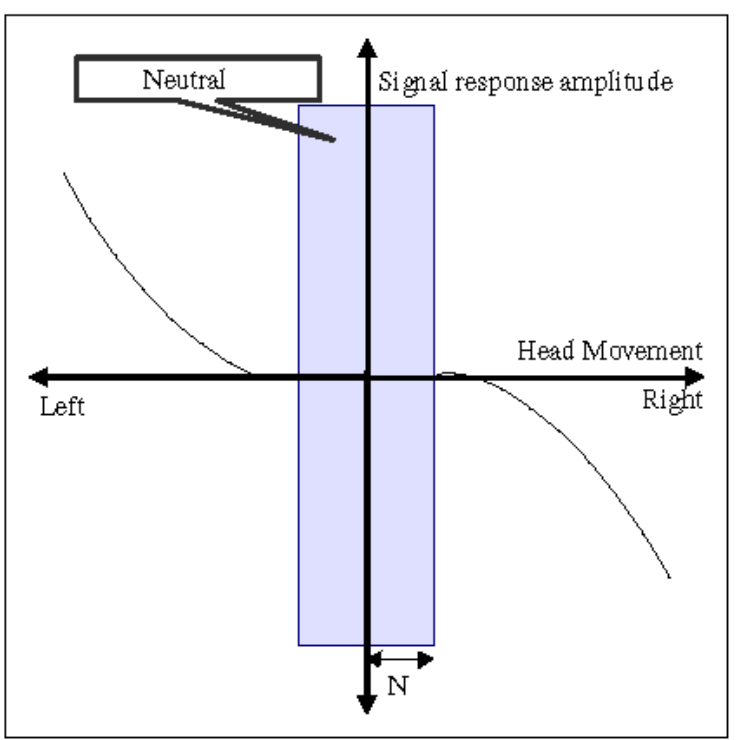

Fig. 10: Mathematical Tracing Function Used in PIC Algorithm

head movements, the car mirror system smoothly changes to provide the driver with the right angles for safe and comfortable driving [14-20].

In comparison to similar systems, our designed and tested system is a simple, cost effective, and accurate positioning system which can easily be interfaces to MCU of any vehicle engine without the need for major modification to the vehicle control system.

\section{REFERENCES}

1. Javad Haddadnia, Karim Faez, and Majid Ahmadi, 2003. An efficient human face recognition system using pseudo zernike moment invariant and radial basis function neural network. Intl. J. Pattern Recognition and Artificial Intelligence, 17: 41-62. 
2. Heisele, B. and T. Koshizen, 2004. Components for face recognition. In: Proceedings of the $6^{\text {th }}$ International Conference on Automatic Face and Gesture Recognition, Seoul, Korea, pp: 153-158.

3. Ivanov, I., B. Heisele and T. Serre, 2004. Using component features for face recognition. In: Proceedings of the 6th International Conference on Automatic Face and Gesture Recognition, Seoul, Korea, pp: 421-426.

4. Xiao, J., J. Chai and T. Kanade, 2004. A closedform solution to non-rigid shape and motion recovery. In Proceedings of the European Conference on Computer Vision.

5. Pengyu Hong, Zhen Wen, Thomas S. Huang, 2002. Real-time speech-driven face animation with expressions using neural networks. IEEE Transactions on Neural Networks, 13: 1.

6. Covell, M., 1996. Eigen-points: Control-point location using principal component analysis. In International Workshop on Automatic Face and Gesture Recognition, pp: 122-127.

7. Cassell, J., C. Pelachaud, N.I. Badler, M. Steedman, B. Achorn, T. Beckett, B. Douville, S. Prevost, and M. Stone, 1994. Animated conversation: Rule-based generation of facial display, gesture and spoken intonation for multiple conversational agents. In Proc. ACM SIGGRAPH'94, pp: 413-420.

8. Li, Y., F. Yu, Y. Xu, E. Chang, H.Y. Shum, 2001. Speech driven cartoon animation with emotions. Proceedings of the $9^{\text {th }}$ ACM Conference on Multimedia, Ottawa, Canada.

9. Reveret, L., G. Bailly and P. Badin, 2000. MOTHER: A new generation of talking heads providing a flexible articulatory control for videorealistic speech animation. In Proceedings of the $6^{\text {th }}$ Intl. Conference on Spoken Language Processing (ICSLP), Beijing, China, Oct. 16-20.

10. Parke, F.I., 1972. Computer generated animation of faces. Proc. ACM National Conference, 1: 451-457.

11. Chen, L.S., 2000. Joint processing of audio-visual information for the recognition of emotional expressions in human-computer interaction. $\mathrm{Ph}$. D. Thesis. University of Illinois at UrbanaChampaign.
12. Dellaert, F., T. Polzin, and A. Waibel, 1996. Recognizing emotion in speech. In Proc. Intl. Conf. on Spoken Language Processing 1996, Philadelphia, PA, USA, October 3-6, pp: 1970-1973.

13. De Silva, L.C., T. Miyasato, and R. Nakatsu, 1997. Facial emotion recognition using multimodal information. In Proc. IEEE Int. Conf. on Information, Communications and Signal Processing (ICICS'97), Singapore, pp: 397-401.

14. Waters, K. and T.M. Levergood, 1999. DECface, An Automatic Lip-Synchronization Algorithm for Synthetic Faces, Digital Equipment Corporation, Cambridge Research Lab. Technical Report CRL 93-4. [20] V. Blanz and T. Vetter, A Morphable Model for the Synthesis of 3D Faces. In Proc. SIGGRAPH'99.

15. Huang, Y., Y.X. Zhu, G.Y. Xu, 1998. Spatialtemporal features by image registration and warping for gesture recognition. IEEE System, Man and Cybernetics'98, San Diego, USA, October, pp: 4498-4503.

16. Huang, Y., G.Y. Xu, Y.X. Zhu, 2000. Extraction of spatial-temporal features for vision-based gesture recognition. J. Computer Sci. Technol., 15: 64-72.

17. Cootes, T.F., C.J. Taylor et al., 1995. Active shape models-their training and application. Computer Vision and Image Understanding, 61: 38-59.

18. Ekman, P. and W.V. Friesen, 1977. Facial action coding system. Palo Alto, Calif.: Consulting Psychologists Press, Inc.

19. Grant, K.W., B.E. Walden and P.F. Seitz, 1998. Auditory-visual speech recognition by hearingimpaired subjects: Consonant recognition, sentence recognition, and auditory-visual integration. J. Acoust. Soc. Am., 103: 2577-2690.

20. Pandzic, I., J. Ostermann, and D. Millen, 1999. User evaluation: Synthetic talking faces for interactive services. The Visual Computer, 15: 330-340. 